\title{
Nuclear Medicine at a Crossroads
}

\author{
Heinrich R. Schelbert \\ Department of Molecular and Medical Pharmacology, David Geffen School of Medicine at UCLA, Los Angeles, California
}

\begin{abstract}
The growth of molecular imaging heightens the promise of clinical nuclear medicine as a tool for individualization of patient care and for improvement of health-care outcomes. Together with greater use of integrated structure-function imaging, clinical nuclear medicine reaches beyond traditional specialty borders into diagnostic radiology and oncology. Yet, there are concerns about the future of nuclear medicine, including progressively declining reimbursement, the competitive advantages of diagnostic radiology, limited translation of research accomplishments to clinical diagnostic imaging and patient care, and an insufficient pool of incoming highly qualified nuclear medicine clinicians. Thus, nuclear medicine views itself as being at a critical crossroads. What will be important is for nuclear medicine to be positioned as the quintessential molecular imaging modality more centrally within medical imaging and for the integration of nuclear medicine with primary care specialties to be driven more by patient needs than by specialty needs. In this way, the full potential of nuclear medicine as an effective and efficient tool for improving patient outcomes can be realized.
\end{abstract}

Key Words: nuclear medicine; economics; trends

J Nucl Med 2011; 52:10S-15S

DOI: 10.2967/jnumed.110.085639

$\mathbf{T}$ he ever-growing body of science in molecular imaging heightens the promise of clinical nuclear medicine as a tool for greater individualization of patient care and for improving health-care outcomes. Together with greater use of integrated structure-function imaging, clinical nuclear medicine reaches beyond traditional specialty borders into diagnostic radiology and oncology. Yet, there are concerns about the future of nuclear medicine, including progressively declining reimbursement, the competitive advantages of diagnostic radiology, limited translation of research accomplishments to clinical diagnostic imaging and patient care, and an insufficient pool of incoming highly qualified nuclear medicine clinicians. Thus, nuclear medicine views itself as being at a critical crossroads.

Received Nov. 8, 2011; revision accepted Nov. 14, 2011.

For correspondence or reprints contact: Heinrich R. Schelbert, Department of Molecular and Medical Pharmacology, David Geffen School of Medicine at UCLA, B2-085J CHS, 650 Charles E. Young Dr. S., Los Angeles, CA 90095. E-mail: hschelbert@mednet.ucla.edu

COPYRIGHT ( 2011 by the Society of Nuclear Medicine, Inc.

\section{NUCLEAR MEDICINE AND MOLECULAR IMAGING: GROWTH WITHOUT BOUNDARIES}

Clinical nuclear medicine as the quintessential clinical molecular imaging specialty has experienced and continues to experience impressive growth. Imaging is no longer confined to diagnosis and risk stratification of disease but contributes to treatment strategies. This especially applies to oncology, in which PET/CT with ${ }^{18} \mathrm{~F}-\mathrm{FDG}$, an indicator of global tumor vitality, has improved the accuracy with which tumors are detected and staged and with which tumor progression or regression in response to treatment is assessed. Other radiotracers complement the diagnostic arsenal; they enable visualization of tumor types that can escape detection by ${ }^{18} \mathrm{~F}-\mathrm{FDG}$, such as liver, prostate, pancreas, and brain tumors. Radioisotopes that do not require on-site cyclotron production, such as ${ }^{68} \mathrm{Ga}$, now used clinically in several European countries, promise to expand the clinical range of PET; peptides labeled with ${ }^{68} \mathrm{Ga}$ have proved effective for uncovering somatostatin receptorexpressing tumors.

Other radiolabeled probes allow characterization of other biologic properties of tumors, such as perfusion; hypoxia; amino acid, nucleoside, and lipid metabolism; and internal cell receptors. PET can also reveal tumor survival strategies. For instance, when cancer cells switch from glucose to glutamine as the dominant energy substrate (1), they may remain undetected by ${ }^{18} \mathrm{~F}-\mathrm{FDG}$ but can be identified with ${ }^{11} \mathrm{C}$ - or ${ }^{18} \mathrm{~F}$-labeled glutamine as radiotracers of glutaminolysis $(2,3)$. Image-based assays of cell surface receptors allow identification of therapeutic targets. In breast cancer, for example, the responsiveness to hormone treatment depends on the status of estrogen receptor expression, which can be assessed with ${ }^{18} \mathrm{~F}$-estradiol and which predicts responses to hormone treatment (4). As another example, the responsiveness to epidermal growth factor receptor inhibitors depends on the degree of epidermal growth factor receptor expression by the tumor and the presence of activating mutations of the tyrosine kinase domain of the epidermal growth factor receptor. In patients with non-small cell lung cancer, uptake of a ${ }^{11} \mathrm{C}$-labeled small-molecule receptor tyrosine kinase inhibitor predicted the response to epidermal growth factor receptor-targeting treatment with the tyrosine kinase inhibitor erlotinib (5).

Treatment target identification with molecular imaging techniques offers new opportunities for clinical nuclear medicine. Vectors used for image-based target identifica- 
tion but with payloads of therapeutic radioisotopes such as ${ }^{111} \mathrm{In},{ }^{90} \mathrm{Y}$, or ${ }^{177} \mathrm{Lu}$ have met with success in the treatment of neuroendocrine tumors; therapeutic payloads can be precisely calibrated for maximum tumor kill but with the least toxic effect on nontarget tissues.

The addition of integrated dual-modality imaging has accelerated the growth of nuclear medicine. Integrated PET/ CT systems facilitate correction for photon attenuation, thereby shortening imaging times and raising patient throughput. Molecular and functional processes can be colocalized with structural features, enhancing the diagnostic information content and accuracy. The dramatic increase in the number of PET/CT units underscores the clinical relevance of structure-function imaging; between 2001 and 2010, the number of PET/CT systems increased in the United States by more than 10-fold, from about 200 to 2,085 $(6,7)$. More than $84 \%$ of all PET studies are now performed with PET/CT systems, as compared with only 19\% in 2003 (8). The arrival of whole-body PET/MRI promises further enhancements of structure-function imaging capability. Besides anatomic localization, features of tissue composition and texture on high-contrast soft-tissue MR images can now be interrogated within the context of cellular and functional processes, leading to more precise delineation of disease-related alterations and, possibly, earlier disease detection.

Advances in molecular imaging have also occurred in cardiology and neurology. Existing probes have been adapted to-or new probes have been developed for the study of - the cardiovascular system; they have generated new knowledge on atherosclerotic vascular disease and on functional and structural substrates of myocardial disease. In the neurosciences, probes are now available for the study of pre- and postsynaptic function and, importantly, for visualizing histomorphologic substrates of neurodegenerative disorders, probes that hold promise for confirming the presence of Alzheimer disease and for uncovering preclinical stages of neurodegeneration.

Basic research in molecular imaging has advanced at an even greater pace. Already-available targeted probes are being redesigned for improved pharmacokinetics, and novel vectors are being constructed and tested, such as liposomes, engineered antibodies, new protein scaffolds, and nanoparticles with images that are ever more target-specific, recorded with optical devices, ultrasound, Raman spectroscopy, or Cerenkov radiation. Thus far confined to the preclinical environment, research with novel targeted probes approaches first-in-human testing. For example, specifically coated biologically inert gold nanoparticles (coated with polyethylene glycol) and targeted to $\alpha_{2} \beta_{3}$ integrin are currently being tested in a small number of melanoma patients (9).

Molecular imaging research is thus growing without boundaries. It now spans an increasing spectrum of imaging devices and uses a growing arsenal of probes targeting cellsurface and intracellular receptors and functional processes. These advances may well be the prologue to the future molecular imaging clinic. Equipped with the entire range of available imaging devices and with a large arsenal of targeted imaging and therapeutic probes, molecular imaging enables selection of the most appropriate and efficacious imaging technology for the most direct and prompt diagnosis of disease, for risk stratification, and for development of an individualized treatment strategy.

\section{ECONOMICS: CLOUDS ON THE HORIZON}

The evolution and growth of clinical nuclear medicine during the past decade have truly been impressive. In 2010 alone, an estimated 18.7 million radionuclide patient examinations (including PET) were performed. Much of this growth is attributed to myocardial perfusion imaging studies, which in 2010 accounted for $56 \%$ of all nuclear medicine imaging examinations $(6,7)$. Most of these cardiac studies were performed by cardiologists in the nonhospital setting. The other major contributor to growth has been PET, predominantly in cancer patients (94\% of all studies). In 2010 (6), the estimated number of PET examinations in the United States had increased from 248,000 in 2001 to 1.744 million, a more than 7-fold increase. Maturation of imaging instrumentation and software for image display and analysis, integrated PET/CT systems, and recognition of PET's clinical value together with Food and Drug Administration approval and reimbursement by the Centers for Medicare and Medicaid Services (CMS) and third-party carriers have been the major drivers. The growing use of PET has been paralleled by an increasing number of PET sites in the United States (690 sites in 2001 and 2,085 sites in 2010).

What, then, is the likelihood of a continued growth of nuclear medicine and, in particular, PET/CT examinations? Because more than $90 \%$ of all PET/CT studies are performed on cancer patients, population statistics and projections predict a growing pool of cancer survivors. The number of cancer survivors is estimated to grow by about 50\% from 2008 to 2020 (from 12 million to 18 million cancer survivors) (10). Importantly, a greater number of cancer survivors will be 65 y or older in $2020(68 \%$, vs. $60 \%$ in 2008), with a greater illness burden due to comorbidities and, consequently, greater health-care needs than younger cancer survivors. Cancer survivors are defined here as patients in the initial phase after diagnosis, the last year of a life phase, or the continuing phase between the initial and the last year of life phases (11). The projected increase in the number of cancer survivors, especially of older ones, is likely to further raise the demand for PET/CT studies for the initial diagnosis; for staging; and, because treatment strategies are likely to become more targeted and individualized, for therapy monitoring and cancer recurrence.

The projected increase in the number of cancer survivors is bound to be associated with increases in the cost of cancer care. Mariotto et al., for example, anticipate a $27 \%$ increase in the cost of cancer care from 2010 to 2020 (11). 
Using current costs for cancer care, the expenditures are predicted to reach $\$ 258$ billion. If adjusted for an annual $2 \%$ cost increase, this amount increases to $\$ 173$ billion. It is doubtful and unlikely that such anticipated increases in expenditures for cancer care, or more generally for total U.S. health care, can be sustained, especially in view of current efforts to reduce the national debt. Comparison statistics of international health-care expenditures indicate that the United States spends more than 2.4 times the amount per person per year on health care that is spent by other developed countries with comparable or even somewhat better health-care outcomes (12). A fee-for-service reimbursement system; overutilization of tests, procedures, and prescription drugs; and aggressive direct-to-consumer and -provider marketing of prescription drugs and imaging tests in a relatively cost-unconscious patient and provider environment are some of the drivers of the disproportionately higher health-care expenditures in the United States (12). In fact, there has been a $70 \%$ increase in overall imaging use between 2000 and 2005, an increase far in excess of the overall increase in physician-provided services (13). Yet, since the enactment of the Deficit Reduction Act in 2005, the growth rate in imaging use has slowed (14). As compared with a $4.1 \%$ compound annual growth rate for noninvasive imaging from 1998 to 2005, the growth rate for use of noninvasive imaging dramatically declined to $1.4 \%$ between 2005 and 2008 (15). The decline was most dramatic for MRI and for nuclear medicine imaging (with an actual $0.2 \%$ annual decrease from 2005 to 2008).

Use of appropriateness criteria for imaging examinations, diminishing reimbursement rates by CMS and third-party carriers, more stringent requirements for preapproval, and more uninsured patients because of high unemployment have negatively affected the use of imaging services. For example, reimbursement for professional services for SPECT myocardial perfusion imaging or for whole-body PET/CT has declined during the past 3 years by as much as $30 \%-40 \%$, which in fact might account to some extent for the slight decline in the number of radionuclide examinations since 2007 (7). In Medicare fee-for-service beneficiaries, for example, overall use rates of nuclear medicine had increased between 1998 and 2005 but actually declined by $0.2 \%$ between 2005 and 2008 (15). Increases in PET/CT use rates had little effect on this trend for nuclear medicine. Moreover, the PET/ CT contribution to the total cost of cancer care is estimated to be relatively low (i.e., 1.23\%) (16). Nevertheless, there is reason to expect further cuts in reimbursement of advanced imaging services, as Medicare has become a target for cutting government expenditures in order to address the national debt and deficit. Such cuts might entail more stringent requirements for prior authorization of high-cost medical services, increases in the equipment use rates with diminished reimbursement for imaging services, or expansion of multipleprocedure payment reduction, that is, lower professional component payments when more than one imaging study is performed during the same imaging session.
Excessive reductions in imaging services as a consequence of a reduction in health-care expenditures may have a negative impact on patient outcomes. Although such a possible effect still remains to be determined, early studies reporting an association between imaging services and patient outcomes suggest such a possibility. For example, hospitals with greater access to imaging services such as CT, MRI, ultrasound, and radiography report lower mortality rates at a cost that trended to be slightly though not significantly higher (17). One might therefore conclude that restricting access to imaging services raises hospital mortality. Clearly, imaging examinations influence patient management. As the National Oncologic PET Registry with a database of more than 40,000 cancer survivors has shown, physicians changed the intended treatment in response to findings by ${ }^{18} \mathrm{~F}$-FDG PET/CT in $38 \%$ of patients on average (i.e., from surgery to chemotherapy, from curative to palliative, or from curative to supportive care or observation only) (18). Also, as recently reiterated (19), better staging of non-small cell lung cancer with PET reduces rates of futile thoracotomy, of invasive surgery, and of inappropriate surgery-effects that are bound to lower cost. It is especially for PET staging of non-small cell lung cancer that existing evidence supports the cost effectiveness of PETguided individualization of treatment (20). Nonetheless, prospective clinical trials are needed to more firmly support the cost effectiveness of PET not only in patients with nonsmall cell lung cancer but also in patients with other types of cancer. If such cost effectiveness is demonstrated conclusively, then PET will offer several advantages as summarized by Mc Manus and Hicks: "eliminating unnecessary diagnostic procedures, prompt selection of optimal treatment, improved radiotherapy targeting, avoidance of futile aggressive treatment and improved quality of life" (19).

\section{NEW PROBES FOR MASS CONSUMPTION?}

There is the question of whether newly developed radioligands that have been tested and validated in singleor multicenter clinical trials will become available for general clinical use. Also, what is the probability that imaging probes designed and evaluated in the basic research environment will be tested in humans and, if found effective, contribute to patient management? Besides ${ }^{18} \mathrm{~F}-\mathrm{FDG}$ and ${ }^{82} \mathrm{Rb}$, no new PET radiotracers have reached the market, because the pipeline for new radiopharmaceuticals began to dry up during the last decade. The Food and Drug Administration, as part of its Critical Path Initiative, established the exploratory investigational new drug in 2007 to facilitate drug development and firstin-human studies (21). Since then, several new commercially sponsored radioligands are undergoing phase II and III clinical trials, such as ${ }^{18} \mathrm{~F}$-labeled myocardial perfusion tracers and $\beta$-amyloid imaging agents. Issues of intellectual property and lack of exclusive commercial rights or of patent rights for radioligands that are developed in academia have discouraged commercial development. More- 
over, novel radiopharmaceuticals with greater target-specific capability, though potentially having a substantial impact on treatment strategies but only in smaller and more well-defined patient populations, are likely to generate lower financial returns, insufficient to meet the initial expenditures on direct discovery, clinical trials (especially phase II and III), and filing (22,23). Investments of typically \$100-200 million for drug development over an 8- to $10-y$ period require annual sales in the range of $\$ 400$ million in order to be commercially viable, an amount that is unlikely to be met by commercial distribution of more specifically targeted imaging agents for a smaller number of patients. There is also concern among industry that radiopharmaceuticals are undervalued relative to their impact on patient management.

Alternate mechanisms and pathways for the delivery of novel radiotracers to patients are needed. These mechanisms reach beyond traditional government funding and involve professional organizations, academia, and industry. One approach is the strengthening of translational research through greater funding, training, and education of future investigators. Another mechanism entails clinical trial networks as already established by professional organizations such as the American College of Radiology and the Society of Nuclear Medicine, which offer support and expertise in the design of clinical trials and in logistics, organization, data management, and statistics, as well as in standardization of imaging devices, image acquisition, and data analysis. The success of the National Oncologic PET Registry (NOPR), sponsored by the Academy of Molecular Imaging and managed by the American College of Radiology Imaging Network, underscores the effectiveness of such mechanisms. The National Oncologic PET Registry required entry of patient data by PET providers and by referring physicians into a registry for CMS, with CMS coverage of registered patients. The findings of PET's effect on physician-intended treatment strategies in this registry of more than 40,000 cancer patients led to an expansion of PET CMS coverage for cancer patients.

One could envision other scenarios and pathways for approval of a new radioligand for clinical use and reimbursement. Academic institutions and large medical centers that develop a new compound could demonstrate its safety and its effectiveness in answering a specific diagnostic question, and limited approval and reimbursement could be granted for a specifically defined diagnostic application in specified patient populations. For example, in patients with suspected brain tumor recurrence but equivocal findings on MRI, ${ }^{18} \mathrm{~F}-3$,4-dihydroxyphenylalanine (FDOPA) PET/CT could be approved and reimbursed for distinguishing between tumor recurrence and necrosis. Results forthcoming from such highly specified limited uses of a new radiotracer would provide additional evidence of its diagnostic efficiency and could serve as a platform for future multicenter clinical trials.

\section{SEPARATE OR UNIFIED: ARE WE READY?}

The use and impact of clinical molecular imaging will depend critically on the training, expertise, and communication skills of the imaging physician and on that individual's ability to integrate with oncologists, cardiologists, or neurologists in order to become an active participant in patient care. Adequate knowledge of molecular biology and its implications for patient care are prerequisites. Such knowledge will enable the imaging clinician to identify key steps of disease-related biologic processes that can be targeted with imaging probes, as well as to define targets of treatment and assess responses. Expertise in imaging techniques should no longer be limited to specialty-defined imaging devices but should encompass the broad range of imaging technologies such as CT, MRI, ultrasound, and, ultimately, optical imaging approaches.

Practicing clinicians, both in nuclear medicine and in diagnostic radiology, will likely acquire these skills through daily interactions with other imaging specialists and direct care providers, that is, oncologists and radiation oncologists. Yet, the question is whether current training requirements are adequate to prepare the incoming imaging clinician. Equally important is the question of whether the current separation between the specialty fields of diagnostic radiology and nuclear medicine is meaningful, justifiable, and in the best interest of patient care. Will there be a future need for a molecular imaging clinician with skills and expertise that reach across the entire spectrum of current imaging devices and of targeted imaging and therapeutic probes?

A survey of nuclear medicine imaging in the United States indicates that only a relatively small fraction of all nuclear medicine imaging studies are performed and interpreted by nuclear medicine specialists. In 2008, more than $50 \%$ of all nuclear medicine studies were interpreted by cardiologists ( $80 \%$ in the nonhospital setting) (8), reflecting the large share of cardiac studies among all nuclear medicine procedures. Radiologists interpret as many as $56 \%$ of all nuclear medicine studies, whereas only $23 \%$ of studies are interpreted by nuclear medicine specialists.

The Accreditation Council for Graduate Medical Education (ACGME) lists 54 accredited nuclear medicine training programs for 2011, with a total of 147 candidates, a number that has remained relatively stable over the past few years, despite a decrease in the number of training programs (i.e., 67 programs in 2001). Given the current 3-y training requirement, it is assumed that approximately 50 trainees will graduate each year. The small pool of graduates is unlikely to meet the needs of nuclear medicine and, especially, of PET/ CT services, given the more than 1.7 million PET examinations performed in the United States each year. Moreover, employment opportunities for nuclear medicine graduates have remained limited, especially in private practice, despite an expressed need by potential employers for additional physicians in nuclear medicine and PET/CT (24). However, as a recent survey of nuclear medicine trainees indicates, only 
$75 \%$ of candidates found employment in their specialty within 6 mo of graduation. Highest among the employment criteria was the ability to provide services and on-call coverage in areas other than nuclear medicine, areas for which nuclear medicine graduates were considered insufficiently prepared.

Specialty boards and related professional organizations have acknowledged the need for additional training and experience for both nuclear medicine and diagnostic radiology trainees. They have opened intersociety dialogues and have established working groups charged with defining future training needs and how best to implement them (24-27). Also, the American Board of Nuclear Medicine has expanded the training requirements in cross-sectional imaging. A rotation of at least 4 mo in diagnostic $\mathrm{CT}$ in an ACGME-approved diagnostic radiology program is now required. Successful completion of this additional training component should be specifically acknowledged by the American Board of Nuclear Medicine by including a statement of competence in cross-sectional imaging (at least as related to PET/CT) in the examination certificate. This additional training experience and certification could improve the professional outlook of nuclear medicine trainees, possibly increasing the pool of future trainees in nuclear medicine.

The reality, however, is that most of the nuclear medicine and, especially, PET/CT services in the United States are and will continue to be provided by diagnostic radiologists, simply because of the large pool of practicing and incoming radiologists. There are currently 187 ACGME-accredited training programs in diagnostic radiology, with an increasing pool of candidates. Compared with about 3,800 trainees in 2000, the pool has increased by $26 \%$ to 4,800 in 2011 . Certification by the American Board of Radiology requires a minimum 4-mo rotation in an ACGME-approved nuclear medicine program, together with didactic lectures and selfstudy, a training experience that is clearly insufficient to raise the level of PET/CT interpretations beyond "PET positive" and "PET negative" or to apply the full potential of nuclear medicine, PET/CT, and radionuclide therapy to patient care. Nonetheless, most radiology residents consider the current amount of training in nuclear medicine adequate (28). Although only one third of residents consider themselves adequately prepared for performing radionuclide treatment in their future practice, $80 \%$ feel competent to perform PET/ $\mathrm{CT}$ independently. However, it is doubtful that this perceived competence will translate into actual competence. Training and experience in radionuclide therapy are even less adequate to prepare candidates for current radionuclide therapies, which, besides radioiodine treatment of thyroid disease, now include radiolabeled antibodies, microspheres, and peptides for tumor treatment.

Larson's excellent essay (29) in this supplement to JNM provides a detailed account of the need for greater experience in both specialty training programs and how this need might be addressed. The proposal is structured along existing specialty training guidelines and honors the cur- rent separation between specialties. Nevertheless, intertwining both training pathways will enable future diagnostic radiology and nuclear medicine clinicians to more appropriately and efficiently use the full potential of function-structure imaging. Ultimately, however, there will be a need for a molecular imaging clinician who combines knowledge and expertise in hybrid, multiplatform imaging; in image-based assays with targeted probes of tissue function and biology; and in identification of disease-specific targets-tasks that are fundamental for imaging and for targeted treatment. The molecular imaging clinician will thus be able to apply the full potential of molecular imaging and molecular therapy to the patient and to individualized treatment strategies and treatment monitoring. The future imaging clinician will have access to the full spectrum of current and future imaging devices, as well as targeted imaging and therapeutic probes.

Training of future molecular imaging clinicians might require development of new training pathways. Components of knowledge and training requirements have, to some extent, already been established (30). Additional educational components are needed, especially for clinical training and for communication and interaction with other clinical specialties and direct care providers. Training and expertise will then enable the molecular imaging clinician to select the most effective and direct approach to diagnosis, staging, risk assessment, and treatment strategy. Such coordination will avoid unnecessary or ineffective diagnostic tests or eliminate redundant diagnostic approaches, thus reducing cost and radiation exposure; and design and select the most effective patient- and disease-specific treatment strategies, with improved clinical outcomes and better quality of life.

\section{THOUGHTS AND CONCLUSIONS}

The growing prevalence of structure-function hybrid imaging, possible competitive advantages of diagnostic radiology, limited human resources, and declining reimbursement rates have indeed brought nuclear medicine as an independent medical specialty to a crossroads. This is a time of unparalleled growth of molecular imaging research, which holds considerable promise for future image-guided patient care. What will be important is for nuclear medicine to be positioned as the quintessential molecular imaging modality more centrally within medical imaging and for the integration of nuclear medicine with primary care specialties to be driven more by patient needs than by specialty needs. In this way, the full potential of nuclear medicine as an effective and efficient tool for improving patient outcomes can be realized.

\section{ACKNOWLEDGMENTS}

The author wishes to thank Caroline Cortez for her skillful assistance in preparing the manuscript. No potential conflict of interest relevant to this article was reported. 


\section{REFERENCES}

1. Dang CV. Glutaminolysis: supplying carbon or nitrogen or both for cancer cells? Cell Cycle. 2010;9:3884-3886.

2. Lieberman BP, Ploessl K, Wang L, et al. PET imaging of glutaminolysis in tumors by ${ }^{18} \mathrm{~F}-(2 S, 4 R)$-fluoroglutamine. J Nucl Med. 2011;52:1947-1955.

3. Qu W, Oya S, Lieberman BP, et al. Preparation and characterization of L-[5- $\left.{ }^{11} \mathrm{C}\right]$-glutamine for metabolic imaging of tumors. J Nucl Med. In press.

4. Dehdashti F, Mortimer JE, Trinkaus K, et al. PET-based estradiol challenge as a predictive biomarker of response to endocrine therapy in women with estrogenreceptor-positive breast cancer. Breast Cancer Res Treat. 2009;113:509-517.

5. Meng X, Loo BW Jr, Ma L, Murphy JD, Sun X, Yu J. Molecular imaging with ${ }^{11} \mathrm{C}$-PD153035 PET/CT predicts survival in non-small cell lung cancer treated with EGFR-TKI: a pilot study. J Nucl Med. 2011;52:1573-1579.

6. 2011 PET Imaging Market Summary Report. Greenbelt, MD: IMV; 2011.

7. 2011 Nuclear Medicine Market Outlook Report. Greenbelt, MD: IMV; 2011.

8. 2008 Nuclear Medicine Market Summary Report. Greenbelt, MD: IMV; 2008.

9. Friedman R. Nano dot technology enters clinical trials. J Natl Cancer Inst. 2011;103:1428-1429.

10. Parry C, Kent EE, Mariotto AB, Alfano CM, Rowland JH. Cancer survivors: a booming population. Cancer Epidemiol Biomarkers Prev. 2011;20:1996-2005.

11. Mariotto AB, Yabroff KR, Shao Y, Feuer EJ, Brown ML. Projections of the cost of cancer care in the United States: 2010-2020. J Natl Cancer Inst. 2011;103: 117-128.

12. Emanuel EJ, Fuchs VR. The perfect storm of overutilization. JAMA. 2008;299: 2789-2791.

13. Iglehart JK. Health insurers and medical-imaging policy: a work in progress. $N$ Engl J Med. 2009;360:1030-1037.

14. Shaw LJ, Min JK, Hachamovitch R, et al. Cardiovascular imaging research at the crossroads. JACC Cardiovasc Imaging. 2010;3:316-324.

15. Levin DC, Rao VM, Parker L, Frangos AJ, Sunshine JH. Bending the curve: the recent marked slowdown in growth of noninvasive diagnostic imaging. AJR. 2011;196:W25-W29.

16. Yang Y, Czernin J. Contribution of imaging to cancer care costs. J Nucl Med. 2011;52(suppl):86S-92S.
17. Lee DW, Foster DA. The association between hospital outcomes and diagnostic imaging: early findings. J Am Coll Radiol. 2009;6:780-785.

18. Hillner BE, Siegel BA, Shields AF, et al. Relationship between cancer type and impact of PET and PET/CT on intended management: findings of the national oncologic PET registry. J Nucl Med. 2008;49:1928-1935.

19. Mac Manus MP, Hicks RJ. How can we tell if PET imaging for cancer is cost effective? Lancet Oncol. 2010;11:711-712.

20. Langer A. A systematic review of PET and PET/CT in oncology: a way to personalize cancer treatment in a cost-effective manner? BMC Health Serv Res. 2010;10:283.

21. VanBrocklin HF. Radiopharmaceuticals for drug development: United States regulatory perspective. Curr Radiopharm. 2008;1:2-6.

22. Nunn AD. Molecular imaging and personalized medicine: an uncertain future. Cancer Biother Radiopharm. 2007;22:722-739.

23. Nunn AD. The cost of bringing a radiopharmaceutical to the patient's bedside [editorial]. J Nucl Med. 2007;48:169.

24. Guiberteau MJ, Graham MM. ACR-SNM Task Force on Nuclear Medicine Training: report of the task force. J Nucl Med. 2011;52:998-1002.

25. Coleman RE, Delbeke D, Guiberteau MJ, et al. Concurrent PET/CT with an integrated imaging system: intersociety dialogue from the Joint Working Group of the American College of Radiology, the Society of Nuclear Medicine, and the Society of Computed Body Tomography and Magnetic Resonance. J Am Coll Radiol. 2005;2:568-584.

26. Delbeke D, Graham M, Royal H, Segall G, Shulkin BL, Ziessman HA. Conjoint statement of the SNM, ACNM, and ABNM on credentialing and delineation of privileges for therapeutic procedures using radiopharmaceuticals. J Nucl Med. 2011;52:323-326.

27. Graham MM, Metter DF. Evolution of nuclear medicine training: past, present, and future. J Nucl Med. 2007;48:257-268.

28. Lucas RG Jr, Jacene H, Harolds J, Barnes L, Maurer A. Nuclear medicine training for radiology residents: needs assessment survey. Acad Radiol. 2007;14:301-305.

29. Larson SM. The Janus project: the remaking of nuclear medicine and radiology. J Nucl Med. 2011;52(suppl):3S-9S.

30. Zinn KR, Anderson CJ, Bradbury M, et al. Components of a curriculum for molecular imaging scientists. J Nucl Med. 2011;52:650-656. 